Regular paper (Biochemistry; Biochemistry of Proteolysis)

\title{
Impaired chemotaxis and cell adhesion due to decrease in several cell-surface receptors in cathepsin E-deficient macrophages
}

Takayuki Tsukuba ${ }^{1}$, Michiyo Yanagawa ${ }^{2}$, Kuniaki Okamoto ${ }^{1}$, Yoshiko Okamoto ${ }^{4}$, Yoshiyuki Yasuda $^{5}$, Keiichi I. Nakayama ${ }^{6}$, Tomoko Kadowaki ${ }^{3}$, and Kenji Yamamoto ${ }^{7}$

From ${ }^{1}$ Department of Dental Pharmacology, Graduate School of Biomedical Sciences, Nagasaki University, Nagasaki 852-8588; Departments of ${ }^{2}$ Fixed Prosthodontics and ${ }^{3}$ Pharmacology, Graduate School of Dental Science, Kyushu University, Fukuoka 812-8582, Japan; ${ }^{4}$ Department of Biochemistry, Daiichi University College of Pharmaceutical Sciences, Fukuoka 815-8511, Japan, ${ }^{5}$ Division of Clinical Cariology and Endodontology, Department of Oral Rehabilitation, School of Dentistry, Health Sciences University of Hokkaido, Hokkaido, 061-0293. Japan;

${ }^{6}$ Department of Molecular and Cellular Biology, Medical Institute of Bioregulation, Kyushu University, Fukuoka 812-8582, Japan; ${ }^{7}$ Proteolysis Research Laboratory, Graduate School of Pharmaceutical Sciences, Kyushu University, Fukuoka 812-8582, Japan

Running title: Decreased surface receptors on cathepsin E-deficient cells

*To whom correspondence should be addressed: Dr. Takayuki Tsukuba Department of Dental Pharmacology, Graduate School of Biomedical Sciences, Nagasaki University, 1-7-1 Sakamoto, Nagasaki 852-8588, Japan

TEL: 81-95-819-7652; Fax: 81-95-819-7655; E-mail: tsuta@nagasaki-u.ac.jp

(C) The authors 2009. Published by Oxford University Press on behalf of the Japanese Biochemical Society. All rights reserved. 


\section{ABBREVIATIONS}

$\mathrm{CatE}^{-/-}$, cathepsin E-deficient; CCR-2, chemokine receptor; FPRs, $\mathrm{N}$-formyl peptide receptors, LAMP, lysosome-associated membrane protein; TLRs, Toll-like receptors; PE, phycoerythin; FITC, fluorescein isothiocyanate; PBS, phosphate buffered saline; MCP, monocyte chemoattractant protein.

\section{SUMMARY}

Cathepsin $\mathrm{E}$ is an endo-lysosomal aspartic proteinase exclusively present in immune system cells. Previous studies have shown that cathepsin E-deficient $\left(\mathrm{CatE}^{-/ /}\right)$mice display aberrant immune responses such as atopic dermatitis and higher susceptibility to bacterial infection. However, the mechanisms underlying abnormal immune responses induced by cathepsin $\mathrm{E}$ deficiency are still unclear. In this study, we found that the cell-surface levels of chemotactic receptors including chemokine receptor (CCR)-2 and $N$-formyl peptide receptors (FPRs), were clearly diminished in $\mathrm{CatE}^{-/-}$macrophages compared with those in wild-type cells. Consistently, chemotaxis of $\mathrm{CatE}^{-/}$macrophages to MCP-1 and $\mathrm{N}$-formyl-methionyl-leucyl-phenylalanine was also decreased. Similar to the chemotactic receptors, the surface expressions of the adhesion receptors CD18 (integrin $\beta_{2}$ ) and $\mathrm{CD} 29$ (integrin $\beta_{1}$ ) in $\mathrm{CatE}^{-/-}$macrophages were significantly decreased, thereby reducing cell attachment of $\mathrm{CatE}^{-/-}$macrophages. These results indicate that the defects in chemotaxis and cell adhesion are likely to be involved in the imperfect function of $\mathrm{CatE}^{-/-}$macrophages. 


\section{INTRODUCTION}

Cathepsin E (EC 3.4.23.34) is an intracellular aspartic proteinase, which is mainly localized in the endosomal/lysosomal compartments within cells (1-3). Since cathepsin E is predominantly expressed in immune-related cells, including macrophages, dendritic cells, and microglia, this enzyme has been speculated to be involved in the proteolysis of endosomal/lysosomal compartments in immune-related cells (4-6). We have previously generated cathepsin E-deficient $\left(\mathrm{CatE}^{-/}\right)$mice that spontaneously developed atopic dermatitis-like skin lesions when reared under conventional conditions but not under specific pathogen-free conditions (7). These results imply that development of this disorder in these mice appeared to be triggered by some environmental factor(s) such as pathogenic microorganisms. Thereafter, we showed that $C a t E^{-/-}$mice exhibit increased susceptibility to infection with bacteria such as Staphylococcus aureus and Porphyromonas gingivalis (8). However, the molecular mechanisms inducing aberrant immune responses in $\mathrm{CatE}^{-/-}$mice remain to be elucidated.

Recently, we demonstrated that peritoneal macrophages derived from $\mathrm{CatE}^{-/-}$mice display a novel type of lysosomal storage disorder characterized by the accumulation of major lysosomal membrane sialoglycoproteins such as lysosome-associated membrane protein (LAMP)-1 and LAMP-2, in the cells with a concomitant elevated lysosomal pH (9). Since an elevated $\mathrm{pH}$ is known to induce abnormal properties of endolysosomal compartments including abnormal processing and targeting of lysosomal proteins $(10,11)$ and abnormal maturation and/or fusion events of the acidic organelles (12), CatE ${ }^{-/-}$macrophages are assumed to show trafficking defects in both membrane and soluble proteins. Indeed, we found the enhanced secretion of soluble lysosomal enzymes by $\mathrm{CatE}^{-/-}$macrophages (9). In addition to soluble proteins, we reported decreased surface levels of Toll-like receptors (TLRs), which are capable of recognizing specific components of microorganisms, in $\mathrm{CatE}^{-/-}$macrophages, further we reported diminished cytokine production by $\mathrm{CatE}^{-/-}$macrophages that are stimulated by specific 
TLR ligands (8). However, no information is available concerning the trafficking defects of receptors other than TLRs on the cell surface of $\mathrm{CatE}^{-/-}$macrophages.

Macrophages express a large number of cell surface receptors, which recognize a wide range of specific endogenous and exogenous ligands (13). Among them, chemotactic receptors play an important role in immune responses (14). Recognition of chemoattractants by chemotactic receptors, which is known as chemotaxis, regulates leukocyte recruitment to sites of inflammation and infection $(15,16)$. The chemotactic receptors belong to the 7-transmembrane-G-protein-coupled receptor family, and trigger various immune responses besides chemotaxis, induce the production of reactive oxygen species, and stimulate the release of proteolytic enzymes from granules, in the case of neutrophils (17). Concerning chemotactic responses, we have more recently reported that bone-marrow derived macrophages from $C a t E^{-/-}$ mice display impaired chemotaxis in responses to a chemokine and a bacterial peptide (18). However, precise mechanisms of the diminished chemotaxis in $\mathrm{CatE}^{-/-}$macrophages remain unknown. In addition to chemotactic receptors, leukocyte recruitment to inflammatory and infectious sites is orchestrated by cell-adhesion molecules (19). Cell-adhesion receptors are surface proteins involved in the binding of cells with other cells or the extracellular matrix (20, 21). The cell-adhesion receptors belong to 4 major families, namely, the immunoglobulin superfamily, the cadherins, the selectins, and the integrins (22). In particular, the integrins, which are heterodimers formed by the two non-covalent linking 2 subunits $\alpha$ and $\beta$, are also involved in various intracellular signaling pathways as well as in cell attachment $(20,23)$. Therefore, it is of importance to investigate whether or not the chemotactic receptors and cell-adhesion receptors on the cell surface of $\mathrm{CatE}^{-/-}$macrophages are decreased.

In this study, we focused on chemotaxis and cell adhesion in Cat ${ }^{-/-}$macrophages, and observed impaired chemotaxis and cell-adhesion ability in them with concomitant decreased surface levels of chemotactic receptors and cell adhesion receptors compared with those in wild-type cells. 


\section{Materials and Methods}

\section{Materials}

RPMI-1640, fluorescein dextran (70,000 MW), and fluorescein conjugated chemotactic hexapeptide $N$-formyl-Nle-Leu-Phe-Nle-Tyr-Lys were purchased from Invitrogen, Japan. Anti-mouse LAMP-1 and LAMP-2 antibodies were purchased from Southern Biotechnology Inc (Birmingham AL USA). Anti-mouse CCR2 monoclonal antibody conjugated with phycoerythin (PE) was purchased from R\& D Systems Inc. Anti-mouse CD18 and CD29 monoclonal antibodies conjugated with fluorescein isothiocyanate (FITC) were purchased from BD Biosciences.

\section{Mice}

Wild-type and $\mathrm{CatE}^{-/}$mice with the C57BL/6 genetic background were used as described previously (9). Animals were maintained under specific pathogen-free conditions according to the guidelines of the Japanese Pharmacological Society. Animals and all experiments were approved by the Animal and Microbiological Research Committee of the Graduate School of Dental Science, Kyushu University. All experiments were performed with age-matched, male CatE ${ }^{-/-}$ mice and wild-type littermates.

\section{Preparation of peritoneal macrophages}

Thioglycolate-elicited peritoneal macrophages were isolated as described previously (9). Briefly, 8-14 week-old mice were peritoneally injected with $4.05 \%$ thioglycolate $(2 \mathrm{ml} / \mathrm{mouse})$. Three and a half days later, peritoneal exudate cells were isolated from the peritoneal cavity by washing with phosphate buffered saline (PBS). The cells were incubated in RPMI 1640 medium supplemented with $10 \%$ penicillin $(50$ units $/ \mathrm{ml})$ and streptomycin $(50 \mu \mathrm{g} / \mathrm{ml})$ at $37{ }^{\circ} \mathrm{C}$ with $5 \%$ $\mathrm{CO}_{2}$. After incubation for $2 \mathrm{~h}$, floating cells were removed by washing 3 times with 
$\mathrm{Ca}^{2+} / \mathrm{Mg}^{2+}$-free PBS. MAC-2-positive macrophages comprised more than $95 \%$ of the isolated cells.

\section{Cell staining and flow cytometry}

The peritoneal macrophage suspension $\left(2 \times 10^{5}\right.$ cells $\left./ 100 \mu \mathrm{l}\right)$ was incubated on ice for $15 \mathrm{~min}$ with primary antibodies appropriately diluted with PBS containing $2.5 \%$ fetal bovine serum (FBS) and $0.01 \% \mathrm{NaN}_{3}$ (buffer A). Samples were preincubated with Fc Block (anti-mouse CD16/CD32 antibody) (BD Pharmigen), and subsequently with specific antibodies or control antibodies conjugated with PE or FITC for 15 min on ice. In case of permeabilization, cells were prepared by treatment with $0.03 \%$ saponin in buffer A for 15 min on ice. Flow cytometric analyses were performed on a Beckman Coulter Epics XL cytometer.

\section{Cell-adhesion assay}

Fibronectin $(20 \mu \mathrm{g} / \mathrm{ml})$ in RPMI medium or untreated medium (uncoated) was added in a 96-well plate, and incubated overnight at $4^{\circ} \mathrm{C}$. The wells were blocked with RPMI medium containing $0.5 \%$ bovine serum albumin (BSA). After washing with RPMI medium containing $0.1 \%$ BSA (washing buffer), peritoneal macrophages $\left(5 \times 10^{5}\right.$ cells) were added to each well and incubated at $37^{\circ} \mathrm{C}$ for 10,20 , or $30 \mathrm{~min}$. Floating or weakly bound cells in the plate were gently removed by filling the wells 3 times with washing buffer. Bound cells were fixed with $4 \%$ paraformaldehyde in PBS for 10 min. After washing, cells were stained with $50 \mu$ of crystal violet $(5 \mathrm{mg} / \mathrm{ml}$ in $2 \%$ ethanol). After washing 3 times with the washing buffer, the stained plate was assayed with a microplate reader at $540 \mathrm{~nm}$.

\section{Chemotactic assay}

Chemotactic assay was performed as described previously (18). Briefly, peritoneal macrophages $\left(2 \times 10^{5}\right.$ cells $\left./ \mathrm{ml}\right)$ were washed, and resuspended in RPMI medium. Migration of cells in response to the medium alone, MCP-1, or fMLP was assessed in a 24-well chemotaxis chamber separated by polyethylene terephthalate membrane (pore size, $8 \mu \mathrm{m}$ ). The macrophages were 
loaded into the upper chambers and tested for chemoattraction to the media alone (RPMI-1640 medium without FBS as a control medium), MCP-1(0.1 nM), or fMLP $(1 \mu \mathrm{M})$. The chambers were incubated at $37^{\circ} \mathrm{C}$ in $5 \% \mathrm{CO}_{2}$ for $90 \mathrm{~min}$. The cells that migrated into the lower wells were fixed with 4\% paraformaldehyde in PBS for $10 \mathrm{~min}$, subsequently stained with May-Giemsa, and then counted by light microscopy.

\section{Endocytosis assay}

Peritoneal macrophages $\left(5 \times 10^{6}\right.$ cells $)$ were incubated with fluorescein dextran $(20 \mu \mathrm{g} / \mathrm{ml})$ in normal RPMI-1640 medium containing 10\% FBS for 30 or 60 min. After washing with PBS containing $1 \mathrm{mg} / \mathrm{ml}$ of BSA, cells were lysed in $50 \mathrm{mM}$ Tris- $\mathrm{HCl}$ buffer $(\mathrm{pH} 8.5)$ containing $0.1 \%$ Triton X-100. The amounts of fluorescence in the cell lysates were measured by spectrofluorometry (Hitachi 650-40) and normalized to the total cell protein levels.

\section{Statistical analysis}

The statistical significance of differences was determined using the Student's $t$ test.

\section{RESULTS}

Impaired chemotaxis and decreased cell-surface levels of chemotactic receptors in $\mathrm{CatE}^{-/-}$ macrophages

During a series of experiments that we performed, we had assumed that the number of thioglycolate-elicited peritoneal macrophages in $\mathrm{CatE}^{-/}$mice was possibly lower than that in the wild-type mice. Therefore, we accurately measured the number of thioglycolate-elicited peritoneal macrophages in the wild-type and $\mathrm{CatE}^{-/}$mice. The number of peritoneal macrophages in the $\mathrm{CatE}^{-/-}$mice was significantly lower than that in the wild-type mice (Fig. 1), suggesting that chemotaxis or other chemotactic factor(s) might be more impaired in the CatE $\mathrm{E}^{-/}$ mice than in the wild-type mice. 
Recently, we reported that chemotactic responses of the bone- marrow- derived macrophages acquired from $\mathrm{CatE}^{-/}$mice were significantly decreased compared with those from wild-type mice, whereas no marked differences were observed in the responses of bonemarrow- derived dendritic cells acquired from wild-type and $\mathrm{CatE}^{-/-}$mice (18). To further investigate the chemotaxis of peritoneal macrophages, we determined the chemotactic responses of wild-type and $\mathrm{CatE}^{-/-}$macrophages to the typical chemokine monocyte chemoattractant protein (MCP)-1. The number of $C a t E^{-/-}$macrophages that migrated from the upper to the lower chamber in response to MCP-1 was lower than that of the wild-type macrophages (Fig. 2). Similar results were observed in experiments with a bacterial-derived chemoattractant, $N$-formyl-methionyl-leucyl-phenylalanine (fMLP) (Fig. 2). These results of peritoneal macrophages in this study were quite consistent with previous results obtained with those of bone-marrow derived macrophages (18). In addition, we have investigated chemotaxis of resident peritoneal macrophages that were not activated by thioglycolate from wild-type and $\mathrm{CatE}^{-/-}$ mice in response to MCP-1 and fMLP. However, the results of resident macrophages were essentially similar to those of thioglycolate-activated macrophages (Data not shown).

To evaluate the cell-surface levels of chemotactic receptors on the wild-type and CatE ${ }^{-/}$ macrophages, we analyzed the surface expression of CCR2, which is the receptor for MCP-1, and $N$-formyl peptide receptors (FPRs), which are the receptors for several $N$-formyl peptides including fMLP, by flow cytometry. As shown in Fig. 3A, CatE $E^{-/-}$cells showed decreased levels of both CCR2 and FPR as compared with wild-type cells. However, this difference in receptor levels was abrogated with permeabilized cells that were pretreated with saponin, which enabled the detection of both intracellular and extracellular expression of proteins, indicating that the total levels of expression of CCR2 and FPRs between the 2 cell types were comparable (Fig. 3B). 


\section{Reduced cell-adhesion ability and decreased cell surface levels of cell adhesion receptors in $\mathrm{CatE}^{-/-}$macrophages}

To further examine the cell-surface levels of receptors other than TLRs and chemotactic receptors, we investigated the surface expressions of cell-adhesion receptors in peritoneal macrophages from wild-type and $\mathrm{CatE}^{-/-}$mice. Integrins are major cell-adhesion receptors; they are heterodimers composed of an $\alpha$ and $\beta$ subunits (23). When we analyzed the

cell-surface expression of CD18 (integrin $\beta_{2}$ chain) on wild-type and Cat ${ }^{-/}$macrophages by flow cytometry, we observed that the surface expression of CD18 was reduced in $\mathrm{CatE}^{-/-}$ macrophages compared with those of the wild-type cells (Fig.4A). Similarly, the surface levels of CD29 (integrin $\beta_{1}$ chain) on $\mathrm{CatE}^{-/-}$macrophages were more decreased than those of wild-type cells (Fig.4A). However, treatment with saponin showed no significant difference in the total expression levels of receptors between wild-type and CatE ${ }^{-/-}$macrophages (Fig. 4B). These results indicate that the surface levels of cell-adhesion receptors were also decreased in $\mathrm{CatE}^{-/-}$ macrophages in spite of the similarity in the total expression levels of receptors between wild-type and $\mathrm{CatE}^{-/-}$macrophages.

To test the adhesion abilities of wild-type and $\mathrm{CatE}^{-/-}$macrophages, we analyzed the attachment of cells to uncoated or fibronectin-coated plastic plates. It is known that both CD18 and CD29 are involved in the interaction of these cells with plastics or fibronectin (24). At an early incubation time of 10 or $20 \mathrm{~min}$, the density of bound $\mathrm{CatE}^{-/-}$macrophages was significantly lower than that of wild-type macrophages (Fig. 5). However, at a longer incubation time of $30 \mathrm{~min}$, there were no marked differences in the cell density between wild-type and CatE $^{-/-}$macrophages (Fig. 5A). When we analyzed the adhesion of macrophages to fibronectin-coated plates, we observed the density of attached $\mathrm{CatE}^{-/-}$macrophages to be diminished more than that of wild-type macrophages at 10 or 20 min of incubation (Fig. 5B). Taken together with the results of the adhesion assay and the flow cytometric analysis, the 
reduced cell-adhesion ability in $\mathrm{CatE}^{-/-}$macrophages is probably due to the decreased surface levels of cell-adhesion receptors in $\mathrm{CatE}^{-/-}$cells.

\section{Decreased cell-surface levels of LAMP-1 and LAMP-2 in CatE ${ }^{-/-}$macrophages}

Our previous study showed that cathepsin E deficiency induces accumulation of LAMP-1 and LAMP-2 in the immune system cells (9). On the other hand, it is known that LAMPs are mainly localized in the limiting membranes of lysosomes and late endosomes; however, small amounts of LAMPs are also detectable on the cell surface $(25,26)$. Therefore, we assumed the possibility that the accumulation of LAMP-1 and LAMP-2 within the cells due to cathepsin E deficiency might cause decreased surface amounts of these lysosomal membrane proteins. We subsequently compared the surface levels of LAMPs between wild-type and CatE ${ }^{-/-}$ macrophages. As shown in Fig. 6, flow cytometric analysis revealed that the surface levels of LAMP-1 and LAMP-2 were notably lower than those of wild-type cells. In contrast, treatment with saponin showed that the total expression levels of $\mathrm{CatE}^{-/-}$macrophages were higher than those of wild-type cells (data not shown). The data are consistent with previous data obtained with western blot or flow cytometric analysis performed to evaluate LAMP-1 and LAMP-2 expression $(9,18)$. Thus, the surface expressions of LAMP-1 and LAMP-2 were also decreased in $\mathrm{CatE}^{-/-}$macrophages, probably because these lysosomal proteins accumulated in endosomes and lysosomes in $\mathrm{CatE}^{-/-}$cells (9).

\section{Normal endocytosis of FITC-dextran in $\mathrm{CatE}^{-/-}$macrophages}

We finally examined whether cathepsin E deficiency might affect the vesicle transport, including endocytosis, in addition to the membrane transport to the cell surface. We subsequently analyzed endocytosis in macrophages with FITC-dextran. After incubation at 30 or $60 \mathrm{~min}$, we measured the intracellular amounts of FITC-dextran in wild-type and CatE $\mathrm{E}^{-/-}$ macrophages by spectrofluorometry. However, there was no notable difference in the endocytic 
efficiency of FITC-dextran between wild-type and $\mathrm{CatE}^{-/-}$macrophages (Fig. 7). We also found no significant difference between the endocytosis of wild-type and $\mathrm{CatE}^{-/-}$macrophages at $24 \mathrm{~h}$ of incubation time (data not shown). Therefore, it appears probable that the cathepsin E deficiency causes membrane trafficking defects, but not vesicle trafficking defects such as endocytosis.

\section{DISCUSSION}

This study indicates that surface levels of CCR2 and FPRs chemotactic receptors, and CD18 and CD29 adhesion receptors were decreased in $\mathrm{CatE}^{-/}$macrophages. Consistent with the reduced levels of chemotactic and adhesion receptors, the chemotactic and cell adhesion ability

were also defective in $\mathrm{CatE}^{-/-}$macrophages. In addition to surface proteins, the surface levels of LAMP-1 and LAMP-2 were diminished in $\mathrm{CatE}^{-/-}$macrophages. However, the endocytosis of dextran in wild-type and $\mathrm{CatE}^{-/}$macrophages was indistinguishable. Thus, the membrane trafficking of some receptors and membrane proteins to the cell surface and their functions were partially defective in $\mathrm{CatE}^{-/-}$macrophages.

Our previous study has shown that cathepsin E deficiency induces accumulation of LAMP-1 and LAMP-2 accompanied by an elevation of lysosomal pH (9). Moreover, we have reported that the surface levels of TLR2 and TLR4 are decreased in $C a t E^{-/-}$macrophages, further we have reported that their cytokine production by $\mathrm{CatE}^{-/}$macrophages in response to the specific TLR2 ligand (peptideglycan) and the TLR4 ligand (lipopolysaccharide) is diminished (8). However, this study demonstrated that surface levels of not only TLR, but also the chemotactic receptors and the adhesion receptors were decreased in $\mathrm{CatE}^{-/-}$macrophages. Importantly this study showed that the surface levels of LAMP-1 and LAMP-2 were also diminished in $\mathrm{CatE}^{-/-}$macrophages. Thus, cell-surface receptors and the surface expression of LAMP proteins are clearly attenuated in $\mathrm{CatE}^{-/-}$macrophages, implying an abnormal membrane trafficking of some proteins in $\mathrm{CatE}^{-/-}$cells. 
The abnormal membrane trafficking in $\mathrm{CatE}^{-/-}$cells is probably responsible for the accumulation of various lysosomal membrane sialoglycoproteins including LAMP-1 and LAMP-2. This is because cathepsin E deficiency induces a novel form lysosomal storage disorder characterized by the accumulation of LAMPs (9). Therefore, these accumulations in endosomes and lysosomes probably influence the defects in the trafficking of membrane proteins in endocytic pathways. Similar abnormal membrane trafficking is observed in other lysosomal storage diseases such as Gaucher disease ( $\beta$-glucosidase deficiency) (27), Pompe diseases ( $\alpha$-glucosidase deficiency) (28), neuronal ceroid lipofuscinosis (CLN3 or battenin deficiency) (29), and Niemann-Pick type A or B disease (sphingomyelinase deficiency) (30). It has been proposed that accumulation of metabolites due to lack of a lysosomal enzyme causes a certain "traffic jam" in the endocytic pathways including those at the cell surface $(31,32)$. Moreover, it has been reported that the treatment of cells with substances that are indigestible by lysosomes, which induces lysosomal storage-like phenotypes, and results in the accumulation of the cell-surface proteins MUC1 and CD29 cell surface proteins in endocytic vesicles (33). Thus, in a manner analogous to these phenotypes of storage disorders, it is likely that cathepsin E deficiency primarily causes accumulation of LAMPs, secondarily leading to the abnormal membrane trafficking of LAMPs and other receptors to the cell surface.

The molecular mechanisms in which cathepsin E deficiency impairs chemotactic responses and cell-adhesion ability remain to be determined. However, several studies have reported that chemotaxis of cells from lysosomal storage diseases are clearly reduced in comparison with that of cells from normal humans. For example, impaired chemotaxis was observed in cells from Gaucher disease (34) and mucolipidosis II (35). Given that cathepsin E deficiency leads to a lysosomal storage-like disorder in macrophages, it is possible that the lack of cathepsin E causes a defective chemotaxis similar to those lysosomal storage diseases.

In contrast to abnormal membrane trafficking to the cell-surface, endocytosis was normally processed in $\mathrm{CatE}^{-/-}$cells. Consistent with the results of this study, the phagocytosis 
of S. aureus in $\mathrm{CatE}^{-/-}$macrophages has been shown to be normal (8). However, compared with the wild-type cells, dendritic cells from $\mathrm{CatE}^{-/-}$mice exhibit enhanced phagocytosis and increased surface expression of the costimulatory molecules CD86, CD80 and CD40 (18). Although the precise mechanisms are still unknown, the discrepancy in phagocytosis and membrane trafficking between macrophages and dendritic cells from $\mathrm{Cat}^{-/-}$mice may be explained by the findings that dendritic cells have a specialized endocytic pathway, constituting specific compartments for antigen presentation such as the major histocompatibility complex II compartments and class II vesicles $(34,35)$.

In conclusion, the present study indicates impaired chemotactic and cell-adhesion ability in $\mathrm{CatE}^{-/-}$macrophages, probably due to the decreased cell surface levels of chemotactic and cell adhesion receptors.

\section{ACKNOWLEDGEMENTS}

This work was supported in part by a Nagasaki University President's Fund Grant, a Grant from Terumo Life Science Foundation (T.T), and a Grant-in-Aid for Scientific Research from the Ministry of Education, Culture, Sports, Science, and Technology of Japan (T.T, K.Y).

\section{CONFLICT OF INTEREST}

None declared.

\section{REFERENCES}

1. Yamamoto, K. (1999) Cathepsin E and cathepsin D in Proteases: New Perspectives (Turk, V., ed.) pp. 59-71 Birkhauser Verlag, Basel, Switzerland

2. Saku, T., Sakai, H, Shibata, Y., Kato, Y., and Yamamoto, K. (1991) An immunocytochemical study on distinct intracellular localization of cathepsin E and cathepsin D in human gastric cells and various rat cells. J. Biochem. 110, 956-964. 
3. Yasuda, Y., Tsukuba, T., Okamoto, K., Kadowaki, T., and Yamamoto, K. (2005) The Role of the cathepsin E propeptide in correct folding, maturation and sorting to the endosome. $J$. Biochem. 138, 621-630

4. Sastradipura, D.F., Nakanishi, H.., Tsukuba, T., Nishishita, K.., Sakai, H., Kato, Y., Gotow, T., Uchiyama, Y., and Yamamoto, K. (1998) Identification of cellular compartments involved in processing of cathepsin E in primary cultures of rat microglia. J. Neurochem. 70, 2045-2056

5. Nishioku, T., Hashimoto, K., Yamashita, K., Liou, S.Y., Kagamiishi, Y., Maegawa, H., Katsube, N., Peters, C., von Figura, K., Saftig, P., Katunuma, N., Yamamoto, K., and Nakanishi, H. (2002) Involvement of cathepsin E in exogenous antigen processing in primary cultured murine microglia. J. Biol. Chem. 277, 4816-4822.

6. Chain, B., M., Free, P., Medd, P., Swwetman, C., Tabor, B., and Terazzini, N. (2005) The expression and function of cathepsin E in dendritic cells J. Immunol. 174, 1791-1800

7. Tsukuba, T., Okamoto, K., Okamoto, Y., Yanagawa, M., Kohmura, K., Yasuda, Y., Uchi, H., Nakahara, T., Furue, M., Nakayama, K. Kadowaki T., Yamamoto K., and Nakayama K.I.., (2003) Association of cathepsin E deficiency with development of atopic dermatitis. $J$. Biochem. 134, 893-902

8. Tsukuba, T., Yamamoto, S., Yanagawa, M., Okamoto, K., Okamoto, Y., Nakayama, K.I., Kadowaki T., and Yamamoto, K. (2006) Cathepsin E-deficient mice show increased susceptibility to bacterial infection associated with the decreased expression of multiple cell surface Toll-like receptors. J. Biochem. 140, 57-66

9. Yanagawa, M., Tsukuba, T., Nishioku, T., Okamoto, Y. Okamoto, K., Takii, R., Terada, Y, Nakayama, KI., Kadowaki, T, and Yamamoto, K. (2007) Cathepsin E deficiency induces a novel form of lysosomal storage disorder in macrophages showing the accumulation of lysosomal membrane sialoglycoproteins and the elevation of lysosomal pH. J. Biol. Chem. 282, 1851-1862 
10. Mellman, I., Fuchs, R., and Helenius, A. (1986) Acidification of the endocytic and exocytic pathways. Annu. Rev. Biochem. 55, 663-700

11. Wu, M.M., Grabe, M., Adams, S., Tsien, R.Y., Moore, H.P., and Machen, T.E. (2001) Mechanisms of $\mathrm{pH}$ regulation in the regulated secretory pathway. J. Biol. Chem. 276, $33027-33035$

12. Weisz, O.A. (2003) Organelle acidification and disease. Traffic. 4, 57-64.

13. Taylor, P.R., Martinez-Pomares, L., Stacey, M., Lin, H.H., Brown, G.D., and Gordon, S. (2005) Macrophage receptors and immune recognition. Annu. Rev. Immunol. 23, 901-944.

14. Mackay, C.R. (2001) Chemokines: immunology's high impact factors. Nat. Immunol. 2, $95-101$

15. Zotnik, A., and Yoshie, O. (2000) Chemokines: anew classification system and their role in immunity. Immunity 12, 121-127

16. Migeotte, I., Communi, D., and Parmentier, M. (2006) Formyl peptide receptors: a promiscuous subfamily of $\mathrm{G}$ protein-coupled receptors controlling immune responses. Cytokine Growth Factor Rev. 17, 501--519.

17. Sallusto, F., Mackay, C. R. and Lanzavecchia, A. (2000) The role of chemokine receptors in primary, effector, and memory immune responses. Annu. Rev. Immunol. 18, 593-620

18. Kakehashi, H., Nishioku, T., Tsukuba, T., Kadowaki, T., Nakamura, S., and Yamamoto, K. (2007) Differential regulation of the nature and functions of dendritic cells and macrophages by cathepsin E. J. Immunol. 179, 5728-5737

19. Luo, B.H., Carman, C.V. and Springer, T.A, (2007) Structural basis of integrin regulation and signaling, Annu. Rev. Immunol. 25, 619-647

20. Harris ES, Prescott SM, McIntyre TM, and Zimmerman, G.A. (2000) The leukocyte integrins. J Biol Chem. 275, 23409-23412

21. Gahmberg, C.G. (1997) Leukocyte adhesion: CD11/CD18 integrins and intercellular adhesion molecules. Curr. Opin. Cell Biol. 9, 643-650 
22. Berton, G., and Lowell, C.A. (1999) Integrin signalling in neutrophils and macrophages. Cell Signal. 11, 621-635

23. Luo, B.H., and Springer, T.A. (2006) Integrin structures and conformational signaling. Curr Opin Cell Biol. 18, 579-586.

24. Werthen, M., Sellborn, A., Källtorp, M., Elwing, H., and Thomsen, P. (2001) In vitro study of monocyte viability during the initial adhesion to albumin- and fibrinogen-coated surfaces. Biomaterials. 22, 827-832

25. Fukuda, M. (1991) Lysosomal membrane glycoproteins. Structure, biosynthesis, and intracellular trafficking. J. Biol. Chem. 266, 21327-21330

26. Eskelinen, E.L., Tanaka, Y., and Saftig, P. (2003) At the acidic edge: emerging functions for lysosomal membrane proteins. Trends. Cell Biol. 13, 137-145.

27. Schmitz, M., Alfalah, M., Aerts J.M., Naim H.Y., Zimmer, K.P. (2005) Impaired trafficking of mutants of lysosomal glucocerebrosidase in Gaucher' s disease. Int. J. Biochem. Cell Biol. $37,2310-2320$

28. Meikle, P.J., Yan, M., Ravenscroft, E.M., Isaac, E.L, Hopwood, J.J., Brooks, D.A. (1999) Altered trafficking and turnover of LAMP-1 in Pompe disease-affected cells. Mol. Genet. Metab. 66, 179-188

29. Fossale, E., Wolf, P., Espinola, J.A., Lubicz-Nawrocka, T., Teed, A.M., Gao, H., Rigamonti, D., Cattaneo, E., MacDonald, M.E., and Cotman, S.L. (2004) Membrane trafficking and mitochondrial abnormalities precede subunit $\mathrm{c}$ deposition in a cerebellar cell model of juvenile neuronal ceroid lipofuscinosis BMC Neurosci. 5, 57

30. Pagano, R.E., Puri, V., Dominguez, M., and Marks, D.L. (2000) Membrane traffic in sphingolipid storage diseases. Traffic. 1, 807-815

31. Simons, K., and Gruenberg, J. (2000) Jamming the endosomal system: lipid rafts and lysosomal storage diseases. Trends Cell Biol. 10, 459-462 
32. Futerman, A.H., van Meer, G. (2004) The cell biology of lysosomal storage disorders. Nat. Rev. Mol. Cell Biol. 5, 554-565

33. Ulloa, F., and Real, F.X. (2003) Benzyl- $N$-acetyl-alpha-D-galactosaminide induces a storage disease-like phenotype by perturbing the endocytic pathway. J. Biol. Chem. 278, $12374-12383$

34. Aker M., Zimran A., Abrahamov, A., Horowitz M., and Matzner Y. (1993) Abnormal neutrophil chemotaxis in Gaucher disease. Brit. J. Haematol. 83, 187-191.

35. Sakaguchi T., KikuchiK., Ito S., and Mikawa H. (1988) Impaired neutrophil chemotaxis in two patients with mucolipidosis II. Act Paediatr Scand. 77, 608-609.

36. Mellman I., and Steinman, R.M. (2001) Dendritic cells: specialized and regulated antigen processing machines. Cell 106, 255-258

37. Boes, M. Cuvillier, A. and Ploegh, H. (2004) Membrane specializations and endosome maturation in dendritic cells and B cells. Trend. Cell Biol. 14, 175-183

\section{FIGURE LEGENDS}

Figure 1. Number of thioglycolate-elicited peritoneal macrophages derived from wild-type or $\boldsymbol{C a t E}^{-/-}$mice. Mice were peritoneally injected with $4.05 \%$ thioglycolate $(2 \mathrm{ml} / \mathrm{mouse})$. Three and half days later, peritoneal exudate cells were isolated from the peritoneal cavity by washing with PBS. The cells were incubated with RPMI medium containing $10 \% \mathrm{FBS}$ at $37^{\circ} \mathrm{C}$ for $2 \mathrm{~h}$, and subsequently washed 3 times with PBS. After removing cells with trypsin, the cell number was determined by light microcopy using Burker-Turk hemocytometer chambers. The data are means \pm SD for five independent experiments. ${ }^{*} P<0.01$, versus the corresponding values for the wild-type littermates, obtained with the unpaired Student's $t$ test. 
Figure 2. Chemotaxis of peritoneal macrophages from wild-type or $\mathrm{CatE}^{-/-}$mice in response to MCP-1 and fMLP. Peritoneal macrophages were washed, and resuspended in RPMI-1640 medium without FBS as a control medium. Migration of cells in response to a concentration of MCP-1 $(0.1 \mathrm{nM})$, and fMLP $(1 \mu \mathrm{M})$ was assessed in a 24-well Transwell chemotaxis chamber. The chambers were incubated at $37^{\circ} \mathrm{C}$ for $90 \mathrm{~min}$. The cells that migrated to the lower well were fixed-, and subsequently stained with trypan blue, and then counted by light microscopy. The data are means $\pm \mathrm{SD}$ for five independent experiments. ${ }^{*} P<0.05$, versus the corresponding values for the wild-type macrophages, obtained with the unpaired Student's $t$ test.

Figure 3. Flow cytometric analysis of expression levels of CCR2 and FPRs in wild-type or $\boldsymbol{C a t E}^{-/-}$macrophages. (A) Peritoneal macrophages $\left(2 \times 10^{5}\right.$ cells $)$ from wild-type and $\mathrm{CatE}^{-/-}$ mice were stained for cell surface with a specific antibody for CCR2 conjugated with PE, or with $N$-formyl-Nle-Leu-Phe-Nle-Tyr-Lys-fluorescein, and then analyzed by flow cytometry. Data are representative of five independent experiments. (B) The cells were permeabilized with saponin, and then stained for determination of the total expression levels of CCR2 or FPRs. Data are representative of 5 independent experiments.

Figure 4. Expression levels of CD18 and CD29 in wild-type or $\mathrm{CatE}^{-/-}$macrophages. (A) Macrophages $\left(2 \times 10^{5}\right.$ cells $)$ from wild-type and $\mathrm{CatE}^{-/-}$mice were stained for the cell surface with specific antibodies for CD18 and CD29, subsequently treated with FITC-labeled second antibodies, and then analyzed by flow cytometry. Data are representative of five independent experiments. (B) The cells were permeabilized with saponin and then stained for determination of the total expression levels of CD18 or CD29. Data are representative 5 of independent experiments. 
Figure 5. Cell adhesion of wild-type and $\mathrm{CatE}^{-/-}$macrophages on uncoated or fibronection-treated plates. Peritoneal macrophages $\left(5 \times 10^{5}\right.$ cells $)$ were added to uncoated or fibronectin-treated plastic wells, and subsequently incubated at $37^{\circ} \mathrm{C}$ for 10,20 or $30 \mathrm{~min}$. After washing, bound cells were fixed with $4 \%$ paraformaldehyde in PBS, and then stained with crystal violet. The stained plates were assayed with a microplate reader at $540 \mathrm{~nm}$. The data are means \pm SD for four independent experiments. ${ }^{*} P<0.05$, versus the corresponding values obtained with the wild-type macrophages, obtained with the unpaired Student's $t$ test.

Figure 6. Surface expressions of LAMP-1 and LAMP-2 in wild-type or CatE ${ }^{-/-}$ macrophages. Macrophages $\left(2 \times 10^{5}\right.$ cells $)$ from wild-type and $\mathrm{CatE}^{-/-}$mice were stained for the cell surface with specific antibodies for LAMP-1 and LAMP-2, subsequently treated with FITC-labeled second antibodies, and then analyzed by flow cytometry. Data are representative of five independent experiments.

Figure 7. Endocytosis of FITC-dextran by wild-type or $\mathrm{CatE}^{-/-}$macrophages. Macrophages $\left(5 \times 10^{6}\right.$ cells $)$ were incubated with FITC-dextran $(20 \mu \mathrm{g} / \mathrm{ml})$ for 30 or $60 \mathrm{~min}$. After washing, cells were lysed in $50 \mathrm{mM}$ Tris- $\mathrm{HCl}$ buffer $(\mathrm{pH} 8.5)$ containing $0.1 \%$ Triton $\mathrm{X}-100$. The amounts of fluorescence in the cell lysates were measured by spectrofluorometry and normalized to the total cell protein concentration. 


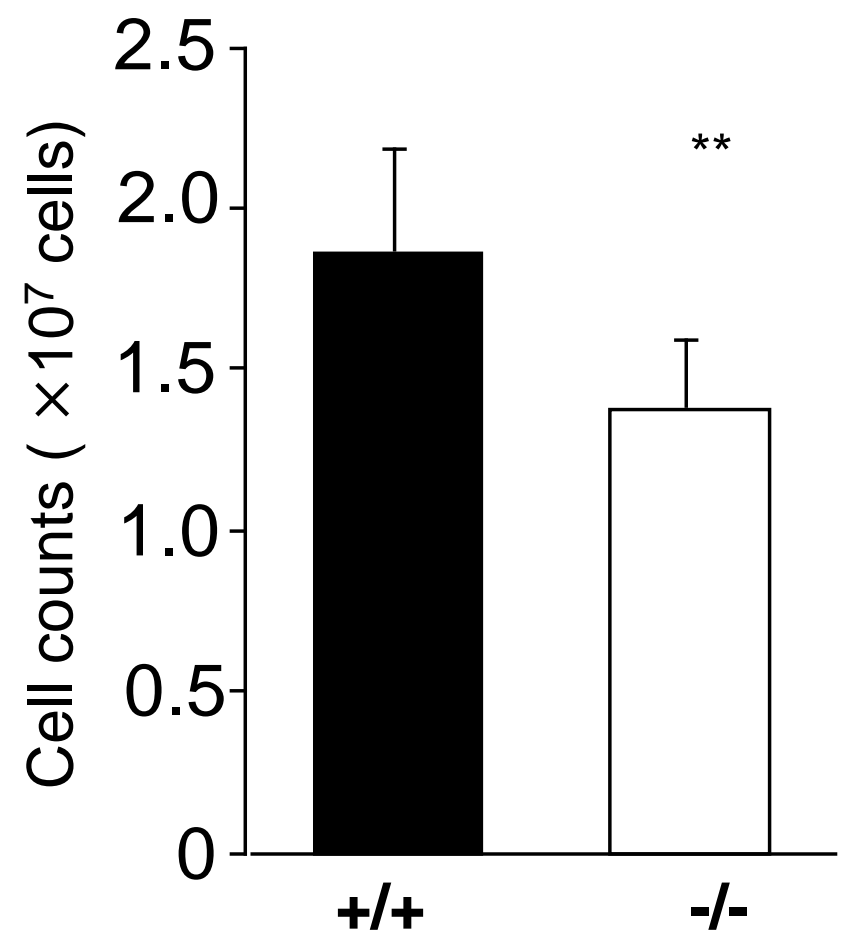

Tsukuba et. al. Figure 1 


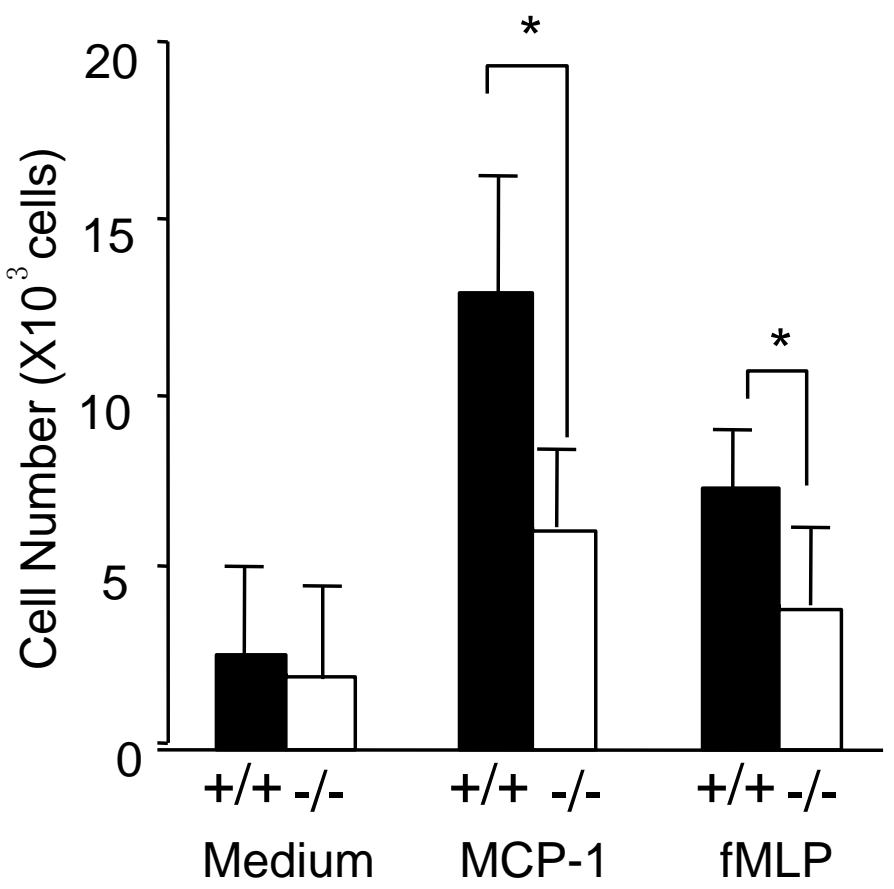

Tsukuba et. al. Figure 2 

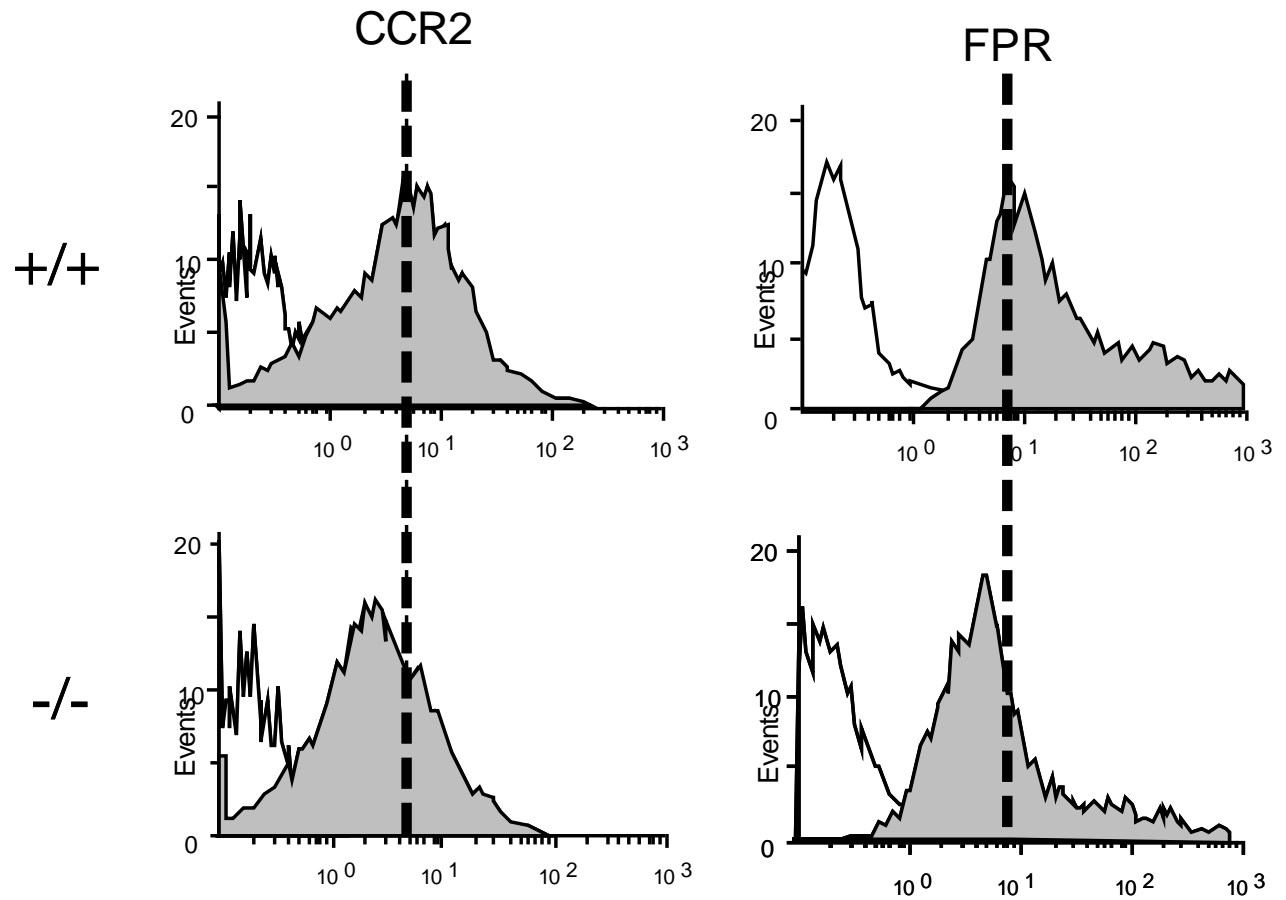

B

Permeabilized

CCR2

FPR
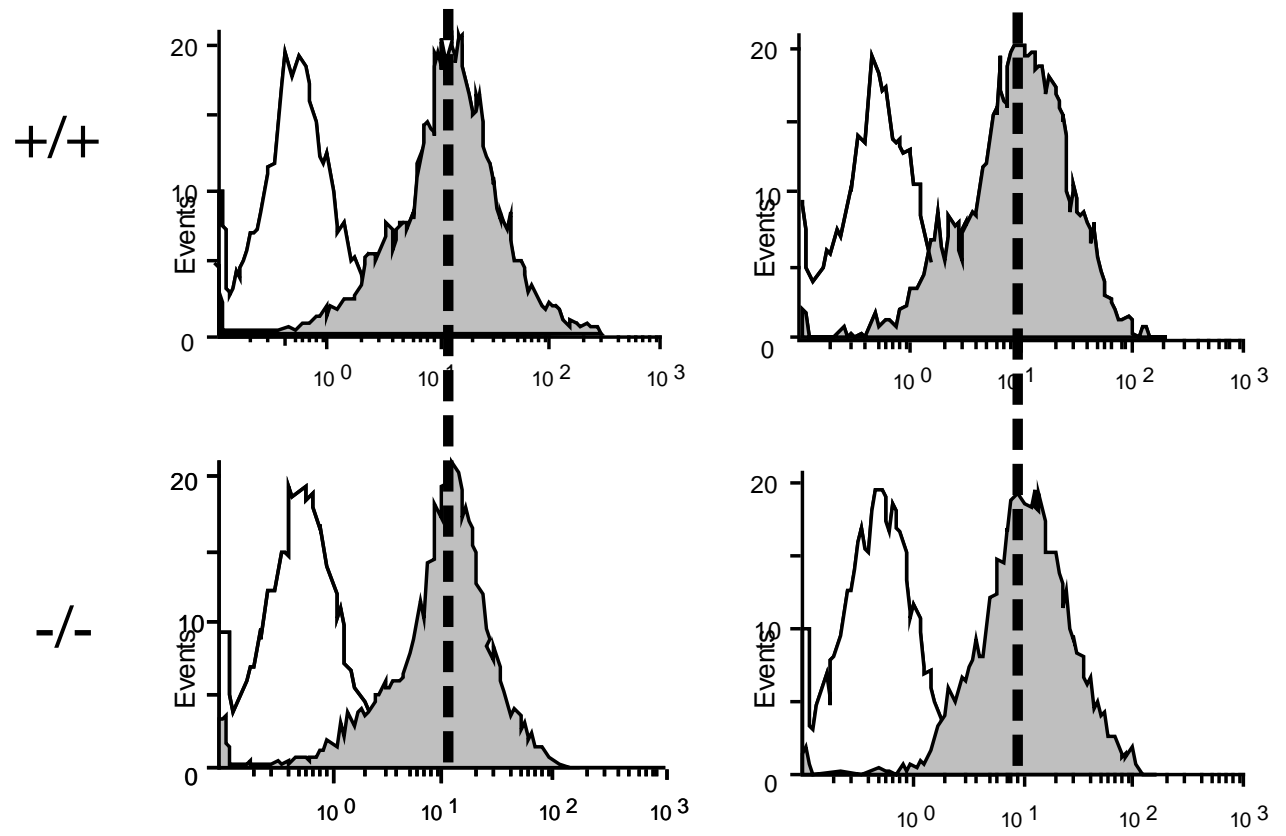

Fluorescence intensity

\section{Tsukuba et. al. Figure 3}


A

Intact

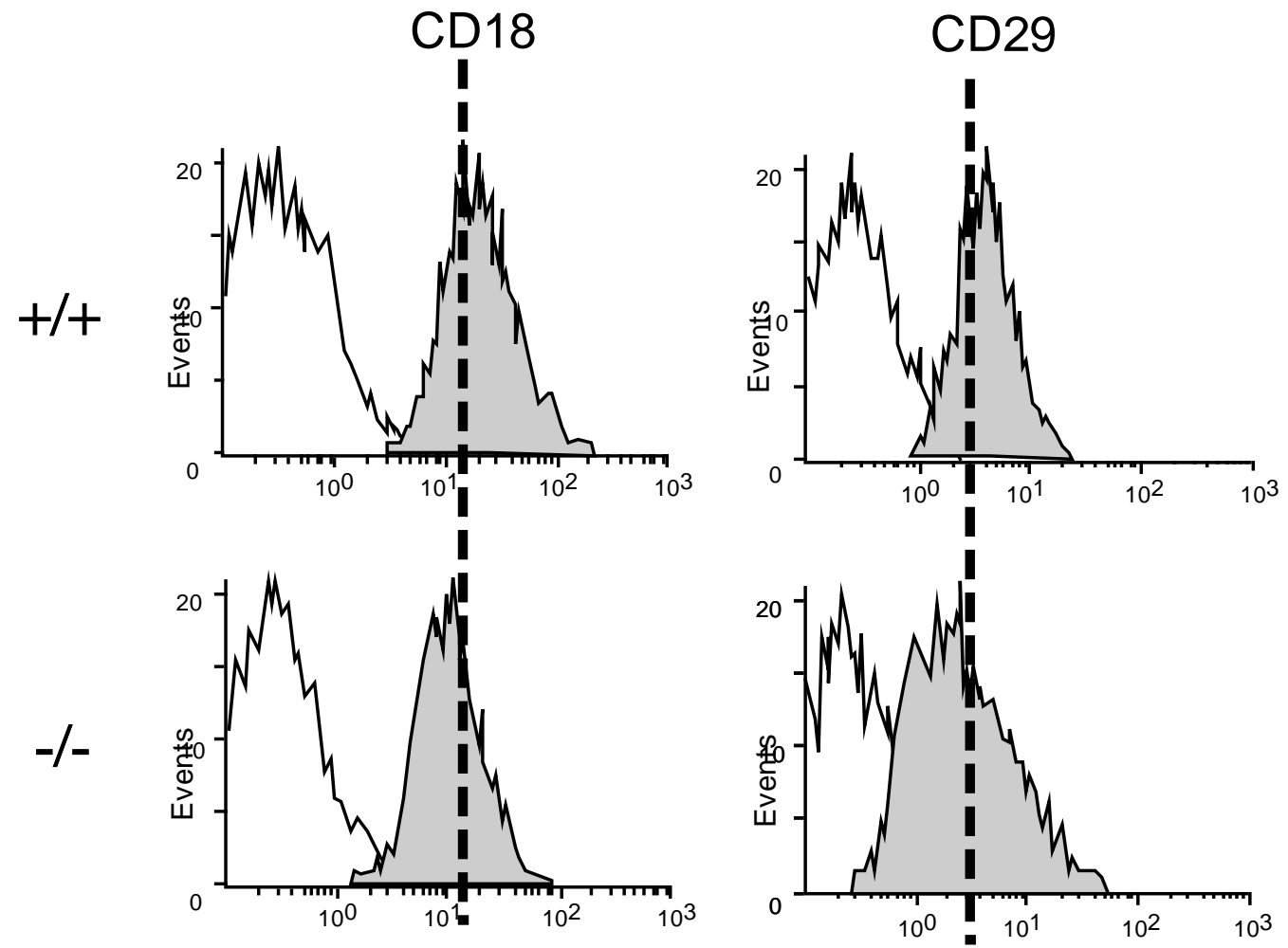

B

Permeabilized
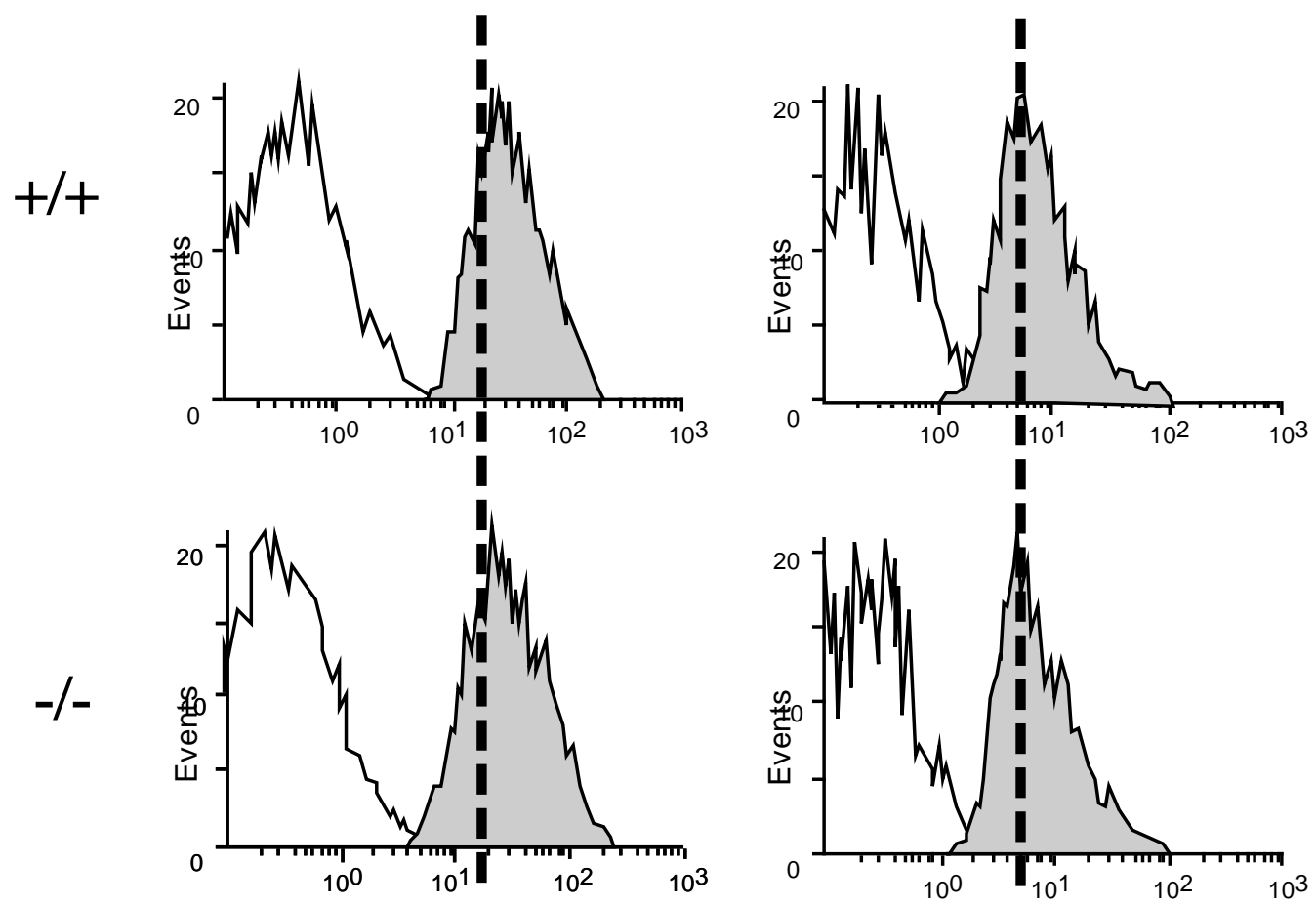

Fluorescence intensity

Tsukuba et. al. Figure 4 
A

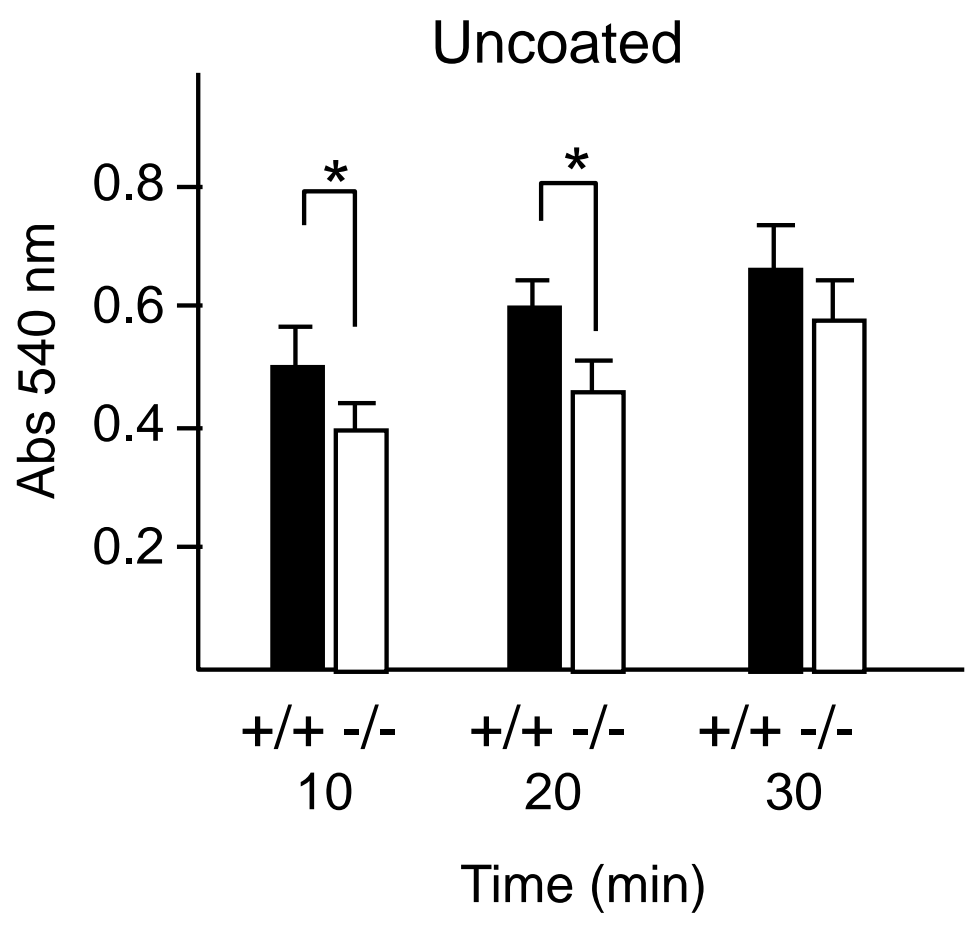

B

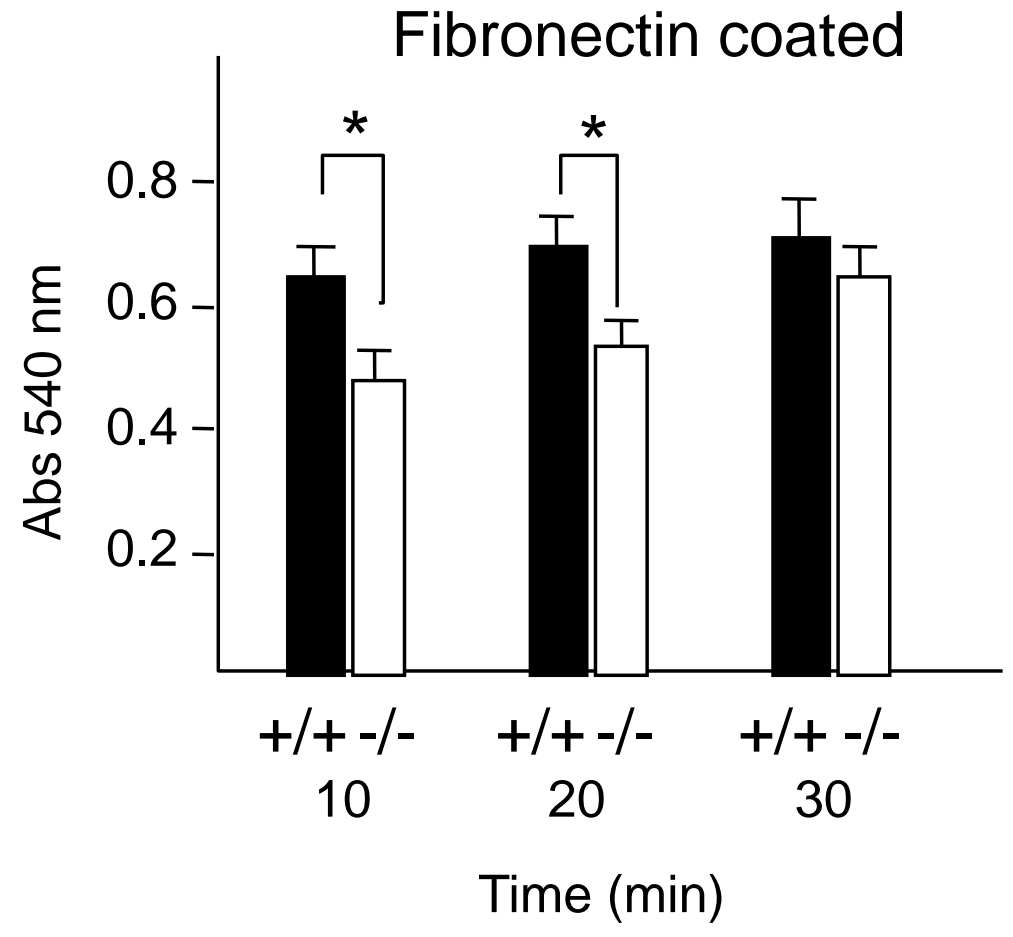

Tsukuba et. al. Figure 5 
Intact
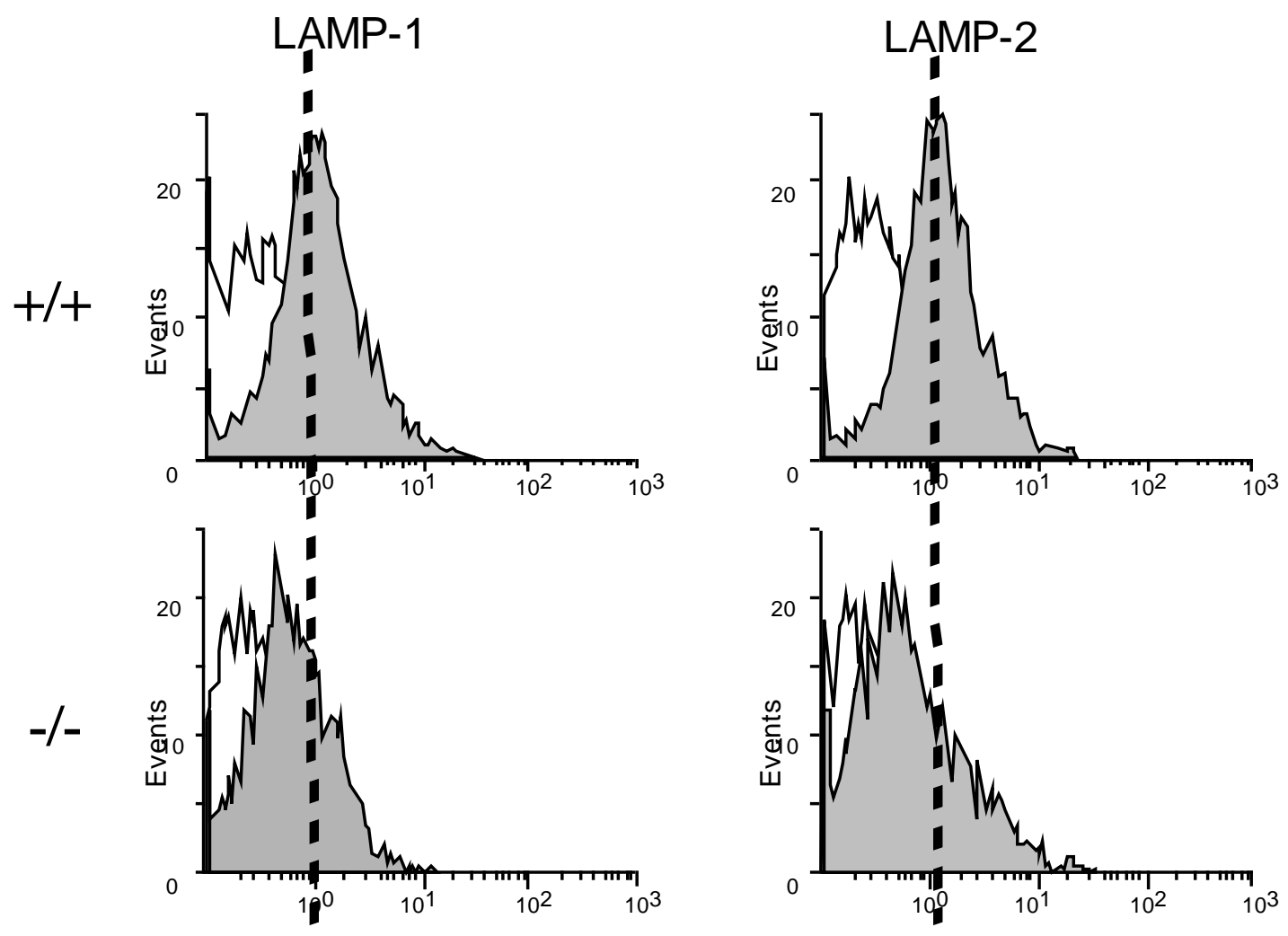

Fluorescence intensity 


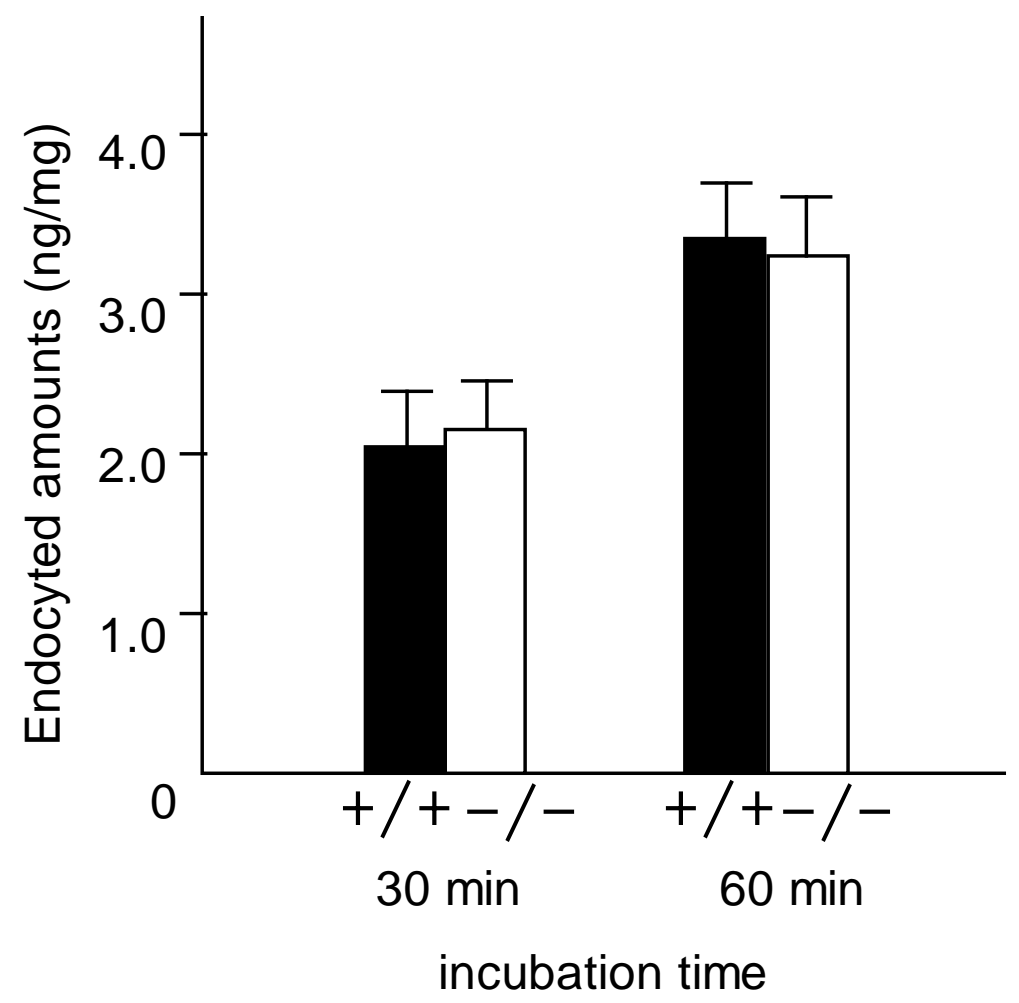

Tsukuba et. al. Figure 7 